\title{
Fluid type and volume reduce risk of post-ERCP pancreatitis and length of hospital stay in high-risk patients: a secondary analysis of the INDIEH trial*
}

\section{다 (1) $(2)$}

\author{
Authors \\ Anthony N. Kalloo², Vikesh K. Singh ${ }^{* * *}, 2$, D. Nageshwar Reddy ${ }^{* * *}, 1$ \\ Institutions \\ 1 Asian Institute of Gastroenterology, Hyderabad, \\ Telangana, India \\ 2 Division of Gastroenterology, Johns Hopkins Medical \\ Institutions, Baltimore, Maryland, United States \\ 3 Apollo Gleneagles Hospital, Kolkata, West Bengal, India \\ 4 Postgraduate Institute of Medical Education \& Research, \\ Chandigarh, India \\ 5 Division of Gastroenterology and Hepatology, Medical \\ University of South Carolina, Charleston, South Carolina, \\ United States
}

Rupjyoti Talukdar ${ }^{* *}, 1$, Ayesha Kamal ${ }^{* *}, 2$, Venkata S. Akshintala², Rajesh Goud ${ }^{1}$, Sundeep Lakhtakia1, Mohan K.

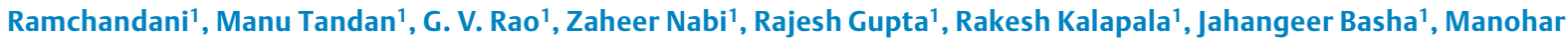
Reddy ${ }^{1}$, Vijay K. Rai ${ }^{3}$, Mahesh K Goenka ${ }^{3}$, Saroj Sinha4, Rakesh Kochhar ${ }^{4}$, B. Joseph Elmunzer ${ }^{5}$, Mouen A. Khashab², submitted 4.9.2019

accepted after revision 23.3.2020

\section{Bibliography}

DOI https://doi.org/10.1055/a-1149-1359 |

Endoscopy International Open 2020; 08: E834-E839

(c) Georg Thieme Verlag KG Stuttgart · New York eISSN 2196-9736

\section{Corresponding author}

Vikesh K. Singh, MD, MSc, Johns Hopkins University School of Medicine, 1830 E. Monument Street, Room 428,

Baltimore, MD 21205

Fax: +1-410-614-7631

vsingh1@jhmi.edu

\section{ABSTRACT}

Background and study aims Impact of intravenous fluid administration on prophylaxis against post-endoscopic retrograde cholangiopancreatography pancreatitis (PEP) has not been rigorously evaluated among patients at highrisk for PEP.

Patients and methods Effect of volume and type of fluid administered on PEP incidence was studied through a secondary analysis of high-risk patients who underwent endoscopic retrograde cholangopancreatography (ERCP) as a part of a randomized controlled trial in which all patients received rectal indomethacin. Periprocedural fluid was defined as fluid infused during and after ERCP.

Results A total 960 patients were randomized during the trial, of whom $476(49.6 \%)$ received periprocedural fluids (mean volume $=1245 \mathrm{~mL}[ \pm 629]$ ). There was a trend towards a lower incidence of PEP in patients who received periprocedural fluid vs. those who did not ( $5.2 \%$ vs. $8.0 \%$, $P=0.079$ ). Among those receiving fluids, those who did not develop PEP received a higher mean volume of fluid vs. who developed PEP $(1012 \pm 725 \mathrm{~mL}$ vs. $752 \pm 783 \mathrm{~mL}, P=$ 0.036). Among 174 patients (37\%) who received LR, patients who did not develop PEP received a higher mean volume of LR vs. those who developed PEP $(570 \pm 559 \mathrm{~mL}$ vs. $329 \pm 356 \mathrm{~mL}, P=0.006)$. Length of hospital stay decreased as the volume of periprocedural volume administration increased $(r=0.16, P<0.001)$.

Conclusion Higher fluid volume and lactated Ringer's use during the periprocedural period was associated with a decreased risk of PEP and length of hospital stay beyond rectal indomethacin in high risk patients.

\footnotetext{
* Meeting presentations: The preliminary data from this study were presented as an oral presentation at the annual meeting of the World Congress of Gastroenterology and American College of Gastroenterology on October 17, 2017 in Orlando, Florida. The study was awarded the ACG Governors Award for Excellence in Clinical Research.
} 


\section{Introduction}

Acute pancreatitis (AP) is the most common complication of endoscopic retrograde cholangiopancretography (ERCP) [1,2]. Since the time of injury to the pancreas is known in patients undergoing ERCP, several pharmacologic agents have been studied for the prevention of post-ERCP pancreatitis (PEP). Rectal indomethacin has been the only drug thus far associated with a significantly lower risk of PEP if given immediately after ERCP [3]. In addition, prophylactic pancreatic duct stent placement has also been shown to significantly reduce the risk of PEP in high-risk patients [4].

Fluid therapy has been the mainstay of treating acute pancreatitis for years in the absence of a specific pharmacological therapy [5]. The role of fluids in PEP was first evaluated by Cote et el. in a retrospective study that showed a decreased length of hospital stay in patients who received increased volumes of fluid in the first 24 hours after undergoing ERCP [6]. Following this, two randomized controlled trials have shown a decreased incidence of PEP among average-risk patients with use of lactated Ringer's (LR) solution [7, 8]. However, both of these trials assessed the impact of fluid administration over 8 hours after ERCP and, therefore, may lack generalizability to centers who perform ERCP in primarily outpatients and/or cannot keep patients in post-anesthesia recovery areas for long periods of time. These trials also did not evaluate concurrent use of lactated Ringer's and rectal indomethacin, which best reflects current clinical practice in most centers [3]. In addition, neither of these trials evaluated high-risk patients. A more recent trial, utilizing a $2 \times 2$ factorial design, evaluated the combination of $L R$ and rectal indomethacin in high risk patients and found a significant difference, in favor of combination prophylaxis, over a pure placebo arm. Many would question the inclusion of a "pure" placebo arm, where high risk patients did not receive any prophylaxis [9]. This trial was also markedly underpowered and there was no significant difference found between combination prophylaxis and rectal indomethacin alone.

The primary aim of the current study was to evaluate the impact of type and volume of intravenous (IV) fluid administered on the incidence of PEP in high-risk patients through a secondary analysis of the INDIEH trial [10]. The secondary aim was to determine if the type and volume of IV fluid administered reduced the length of hospital stay.

\section{Patients and methods}

\section{Study design}

The current study is a secondary analysis of a prospective multicenter, double blinded, randomized trial in high risk patients comparing the efficacy of rectal indomethacin versus a combination of topical spray of epinephrine and rectal indomethacin for the prevention of PEP in high-risk patients (INDIEH trial) [10]. The trial was conducted at four tertiary care teaching hospitals, the Johns Hopkins Medical Institutions (United States), Asian Institute of Gastroenterology (India), Postgraduate Institute of Medical Education \& Research (India) and Apollo Gleneagles Hospital (India). Informed consent was obtained from each eligible patient prior to ERCP. An independent Data and Safety Monitoring Board (DSMB) including a group of four experts in ERCP, two from the United States and two from India, reviewed the results of the interim analysis of the INDIEH trial as well as adverse events and provided regulatory oversight.

\section{Study procedure}

The INDIEH trial included adult ( $>18$ years of age) patients who were randomized to receive either a combination of $100 \mathrm{mg}$ of indomethacin and topical spray of $20 \mathrm{~mL}$ of normal saline (indomethacin alone group) or a combination of $100 \mathrm{mg}$ of indomethacin and topical spray of $20 \mathrm{~mL}$ of $0.02 \%$ epinephrine (combination group) at the end of ERCP using a web-based central randomization system, REDCap (research electronic data capture). [11] Only high risk patients meeting one major or two minor validated patient or procedural risk factors for developing PEP were included [3]. Patients who underwent planned therapeutic pancreatic stenting and those with suspected sphincter of Oddi dysfunction (SOD) type 3 were excluded from the study.

Demographics, patient and procedure related risk factors, and follow-up data were recorded on standardized case report forms by the study coordinator at each site who was blinded to allocation assignments of the patients. All data were subsequently entered into REDCap, which was monitored on a weekly basis for completeness and accuracy.

\section{Fluid administration}

Data regarding fluid administration during and after ERCP were collected on all patients in prospective observational manner. Periprocedural fluid was defined as the fluid infused during ERCP in the endoscopy suit and after ERCP in the post-anesthesia care unit (PACU) for up to 6 hours. The volume and type of periprocedural fluid(s) administered was recorded by the study coordinator, while the patient was in the endoscopy suite and/ or PACU. Fluid administration was not determined a priori or controlled for in the INDIEH trial. The use, type and volume of fluid administered was left to the the discretion of the endoscopist, anesthesiologist and/or clinicians in the endoscopy suite and /or PACU.

\section{Study outcomes}

The primary outcome of interest was an evaluation of the impact of volume and type of fluid administration on the incidence of PEP. PEP was defined by the consensus guidelines as 1) New or increased abdominal pain that was clinically consistent with acute pancreatitis; 2 ) Amylase and/or lipase $\geq 3 \times$ the upper limit of normal 24 hours after the procedure; and 3) Hospitalization or prolongation of existing hospitalization for at least 2 days [12]. The secondary outcome was to evaluate impact of fluid administration on the length of hospital stay after ERCP.

\section{Adverse events}

Complications related to ERCP or study interventions including bleeding, perforation and infection, acute renal failure, evidence of overt gastrointestinal bleeding, indomethacin allergy, hypertension and arrhythmias due to epinephrine were record- 
Patients randomized in INDIEH trial $(n=960)$

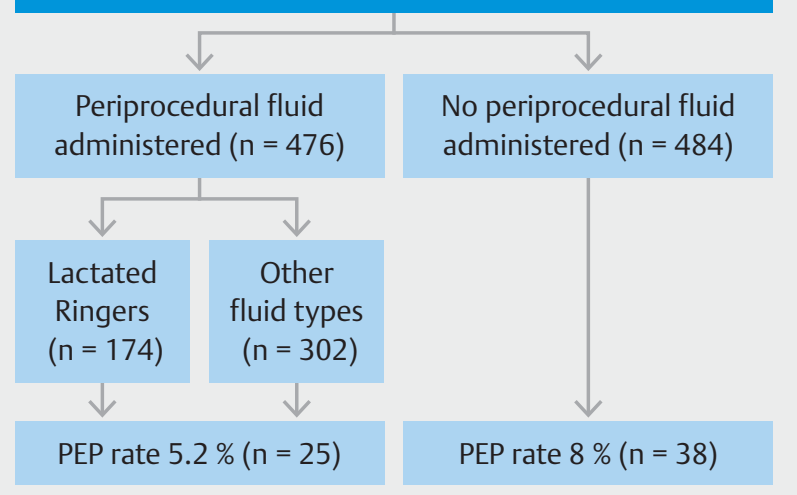

- Fig. 1 Flowchart demonstrating risk of post-ERCP pancreatitis in individual hydration categories ( $P E P=$ post-ERCP pancreatitis).

ed as adverse events (AEs) [12-14]. AEs related to volume overload (development of peripheral edema, pulmonary rales, or ascites) were also recorded. All recorded AEs were reported to the local IRB at each site as well as the DSMB for the trial.

\section{Statistical analysis}

Statistical analysis was performed using SPSS 25 statistical software package (IBM Analytics, Armonk, New York, United States). Categorical variables were assessed by univariable analysis using the Pearson's Chi Square test or Fisher's exact tests as appropriate. Continuous variables were compared using student's $t$ test. Periprocedural fluid therapy and the type of peri- procedural fluid(s) were evaluated for their impact on the incidence of PEP using multivariable regression analysis. Results were expressed as mean \pm standard deviation (SD) and odds ratios ORs with the corresponding $95 \%$ confidence intervals (Cls). A two-sided $P<0.05$ was considered statistically significant. We used scatter plot to show the association between fluid administration and length of hospitalization and performed Pearson test for correlation. This study was registered with ClinicalTrials.gov, number NCT02116309 and data entry into the website was completed at the end of the study [15].

\section{Results}

\section{Baseline characteristica}

A total of 960 patients were enrolled in the study from November 2014 to November 2016 (mean age: $52.33 \pm 14.96$ years, $551(57.4 \%)$ females). Females $<50$ years of age $(25.4 \%)$ and difficult cannulation $(84.9 \%)$ were the most common patient and procedural risk factors, respectively. Trainees were involved in $24 \%$ of the cases.

\section{Fluid administration}

A total of 476 (49.6\%) patients received periprocedural fluids ( $\triangleright$ Fig. 1). The type of fluid included normal saline (NS), dextrose 5\% (D5) and lactated Ringer's (LR). Among the patients who received periprocedural fluids, the volume ranged from $100 \mathrm{~mL}$ to $3000 \mathrm{~mL}$ with a mean of $1245 \mathrm{~mL}( \pm 629)$. There were 174 patients (37\%) who received lactated Ringer's (LR) with a mean of $588 \mathrm{~mL}( \pm 315)$. Patients who were female, whose procedures involved trainees, who were inpatients and who have had a history of post-ERCP pancreatitis received more fluid ( $\bullet$ Table $\mathbf{1})$.

- Table 1 Comparison of baseline characteristics and procedural maneuvers between patients who did and did not receive periprocedural fluids.

\begin{tabular}{|c|c|c|c|}
\hline Patient and procedural characteristics & No periprocedural fluids $(n=484)$ & Received periprocedural fluids ( $n=476$ ) & $P$ value \\
\hline Age-year \pm S.D. & $53.3 \pm 15.8$ & $51.4 \pm 14$ & 0.05 \\
\hline Female gender $-\mathrm{n}(\%)$ & $250(51.7 \%)$ & $298(63.1 \%)$ & $<0.001$ \\
\hline Inpatient status $-\mathrm{n}(\%)$ & $102(21.1 \%)$ & $182(38.2 \%)$ & $<0.001$ \\
\hline $\begin{array}{l}\text { Clinical suspicion of sphincter of Oddi } \\
\text { dysfunction (Type1/2) - n (\%) }\end{array}$ & $7(1.4 \%)$ & $8(1.8 \%)$ & 0.76 \\
\hline History of recurrent pancreatitis - $\mathrm{n}(\%)$ & $4(0.8 \%)$ & $12(2.5 \%)$ & 0.05 \\
\hline History of post-ERCP pancreatitis - $\mathrm{n}(\%)$ & $0(0 \%)$ & $6(1.3 \%)$ & 0.01 \\
\hline Difficult cannulation (>5 attempts) $-\mathrm{n}(\%)$ & $417(86.2 \%)$ & $394(83.5 \%)$ & 0.25 \\
\hline Precut sphincterotomy $-\mathrm{n}(\%)$ & $109(22.5 \%)$ & $89(18.9 \%)$ & 0.41 \\
\hline Pancreatography $-\mathrm{n}(\%)$ & $4(0.8 \%)$ & $8(1.7 \%)$ & 0.26 \\
\hline Pancreatic sphincterotomy $-\mathrm{n}(\%)$ & $6(1.2 \%)$ & $9(1.9 \%)$ & 0.45 \\
\hline Pancreatic acinarization $-\mathrm{n}(\%)$ & $0(0 \%)$ & $2(0.4 \%)$ & 0.24 \\
\hline Trainee involvement $-\mathrm{n}(\%)$ & $33(6.8 \%)$ & $196(41.5 \%)$ & $<0.001$ \\
\hline Topical spray of epinephrine $-\mathrm{n}(\%)$ & $248(51.2 \%)$ & $227(48.1 \%)$ & 0.35 \\
\hline
\end{tabular}




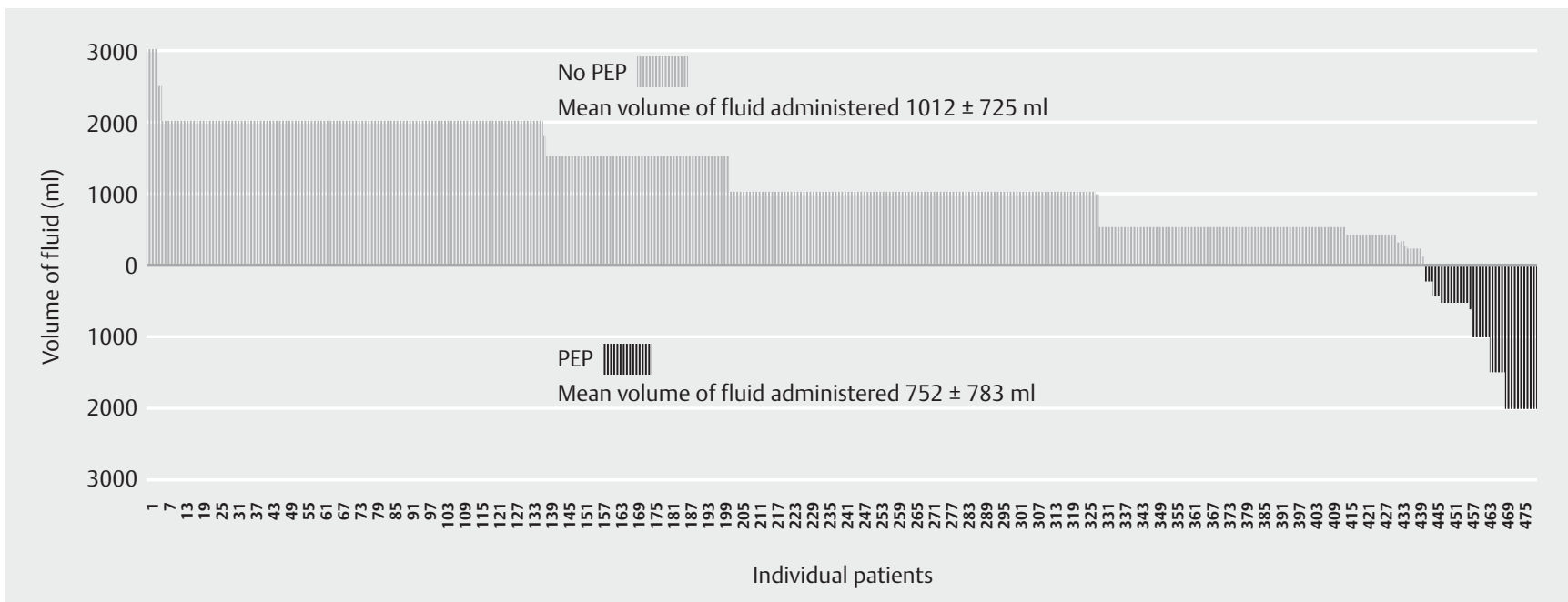

Fig. 2 Waterfall plot displaying the volume of fluid received by patients who developed post-ERCP pancreatitis (PEP) (black bars) and those who did not develop PEP (grey bars).

\section{Study outcomes}

Volume of fluid

There was a trend towards a lower incidence of PEP among patients who received periprocedural fluid compared to those who did not receive periprocedural fluid $(5.2 \%(n=25)$ vs $8.0 \%$ $(\mathrm{n}=38), P=0.079 ;$ OR: $0.65 ; 95 \% \mathrm{Cl} 0.38-1.09)$. Among the 476 patients who received fluids, those who developed PEP received a lower mean volume of fluid $(752 \pm 783 \mathrm{~mL})$ compared to those who did not develop PEP $(1012 \pm 725 \mathrm{~mL}),(P=0.036)$. The distribution of volume of fluid across these two groups is depicted as a waterfall plot ( $\mathbf{F i g . 2}$ ). On the multivariable analysis, after adjusting for all significant variables found on univariable analysis, periprocedural fluids did not significantly impact the incidence of PEP. There was a reduction in the length of hospital stay as the volume of periprocedural fluid administration increased $(r=0.16, P<0.001)(\triangleright$ Fig. 3 ).

\section{Type of fluid}

Patients who developed PEP received a lower volume of LR compared to those who did not develop PEP $(329 \pm 356 \mathrm{~mL}$ vs. $570 \pm 559 \mathrm{~mL}, P=0.006)$. This was, however, not significant on multivariable analysis when adjusted for all variables found significant on univariable analysis. There was a lower trend of PEP among patients who received LR compared to those who received all other fluid types (OR, 0.56 ; $95 \% \mathrm{Cl} 0.31-0.99$ ).

\section{Adverse events}

There were no reports of perforation, infection, myocardial infarction, cerebrovascular accident, acute renal failure or allergic reaction. Potentially attributable adverse events to the study interventions such as post-procedure hypertension (7.05\% vs $7.33 \%, P=0.59$ ), post-sphincterotomy bleeding ( 0 vs $0.2 \%, P=0.36)$, arrhythmia after $\operatorname{ERCP}(0.2 \%$ vs $0, P=0.36)$ were not significantly different between the indomethacin alone and combination groups, respectively. The overall mortality was 0.6 $\%$ and this was unrelated to the primary outcome. There were 6

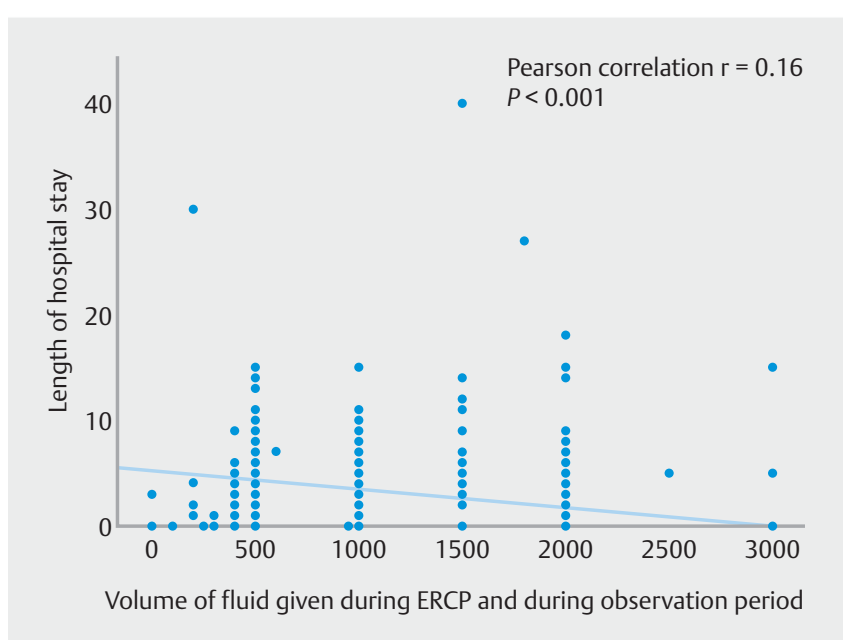

Fig. 3 Scatter plot displaying the volume of fluid received by patients in relation to length of hospital stay.

deaths evenly distributed between the indomethacin alone and combination groups. Four of the deaths were due to metastatic cholangiocarcinoma and two deaths were due to decompensated cirrhosis.

\section{Discussion}

We found that higher mean volumes of IV fluid and use of lactated Ringer's solution was associated with a lower risk of PEP in a secondary analysis of an international multicenter PEP prevention trial in high risk patients. We also found that patients who received increased volumes of fluid had a shorter length of hospital stay.

A few retrospective studies initially showed that higher periprocedural fluid volumes reduce the length of hospital stay after ERCP $[6,16]$. Buxbaum et al. conducted the first RCT 
showing that aggressive hydration is beneficial for preventing PEP in average risk patients [7]. However, this was a pilot study which included only inpatients and was underpowered. Two relatively larger subsequent RCTs showed similar results $[8,17]$. The previous RCTs administered fluids in patients over 8 hours after ERCP which is a longer PACU recovery period than usual when compared to most centers around the world [7,8]. The current multicenter FLUYT trial from the Netherlands has a similar limitation as fluids are administered over 8 hours after ERCP [18]. The results of our study are; therefore, more clinically generalizable across global endoscopic practice as we recorded fluids administered to patients during usual recovery times after ERCP.

Our study further showed that aggressive resuscitation with lactated Ringer's (LR) might lead to a lower incidence of PEP in high-risk patients as compared to administration of other fluid types. This has been supported by previous retrospective as well as prospective studies in average and high risk patients $[7,8,17]$. Mok et el. showed that the combination of LR and indomethacin reduces the risk of PEP from $21 \%$ to $6 \%$ in high-risk patients, when compared to a combination of normal saline and placebo [9]. However, the two by two factorial design of this trial included a placebo arm where normal saline and a placebo suppository were administered to high-risk patients which raises ethical concerns as rectal nonsteroidal anti-inflammatory drug administration has become a common clinical practice for the prevention of PEP in high-risk patients. A clinically more pragmatic trial design would have compared the combination of $L R$ and rectal indomethacin to rectal indomethacin alone. Nonetheless, the trial by Mok found a comparable incidence of PEP in the arm of the study where the combination of indomethacin and LR were administered when compared to the present study. An important downside to the use of large volumes of NS in patients with acute pancreatitis is the development of hyperchloremic acidosis that favors the inflammatory cascade as compared to LR which directly inhibits macrophages involved in inflammation $[19,20]$. Because LR is widely available, safe, and inexpensive and fluids are universally administered to patients undergoing ERCP, infusion of LR represents a simple and convenient option for the prevention of PEP [21].

Our study has several strengths. This is the largest prospective study to date evaluating the effect of fluid administration during and after ERCP in high risk patients using the results of a large multi-center, international randomized controlled trial. As mentioned previously, the results of our study are more generalizable as fluid administration was recorded during the recovery period that would be most commonly observed in outpatients undergoing ERCP. Moreover, half of our patients did not receive any fluids which reflects clinical practice in many countries around the world. Given the trend towards lower PEP rates in patients receiving fluids compared to no fluid administration, our study will hopefully urge more centers around the world to consider incorporating the use of fluids in high risk patients undergoing ERCP. All the risk factors for PEP were adequately represented in our study cohort [10]. We also excluded SOD Type 3 patients, which increases the clinical generalizabil- ity of our study in the post-EPISOD era as compared to the Mok trial where approximately $20 \%$ of patients had SOD.

However, there were several limitations. Our results were not significant on multivariable analysis when adjusted for patient and procedural characteristics which may be due to multiple reasons. First, our patients were not randomized on a priori basis to any specific type or volume of IV fluids as much of the literature supporting fluid administration in patients undergoing ERCP was published after the initiation of our trial. Second, our sample size was not powered to show the independent effect of fluid type and volume on the incidence of PEP. There was a wide range of IV fluid volumes administered which reduces the generalizability of the current study but this may also reflect different fluid administration practices across different centers and countries, thereby providing pragmatic evidence for endoscopists in clinical settings. For example, inpatients were more likely to receive periprocedural fluids compared to those undergoing ERCP on an outpatient basis.

\section{Conclusion}

In conclusion, higher volumes of fluid and LR were associated with further reduction in incidence of post-ERCP pancreatitis and length of hospital stay beyond rectal indomethacin based on this subgroup analysis of a PEP prophylaxis trial of high-risk patients. This analysis was limited due to heterogeneity in the volume of fluid administered since the fluid type or volume was not controlled for in the initial trial. Further adequately powered RCTs are needed to evaluate the effect of type and volume of periprocedural fluids on the development of PEP.

\section{Acknowledgements}

This trial was supported through a 2014 Endoscopic Research Award from the American Society for Gastrointestinal Endoscopy (V.K.S., V.A.). The sponsor funding the study had no role in study design, data collection, data analysis, data interpretation, or writing of the report.

\section{Competing interests}

Dr. Khashab is an advisory board member and consultant for Boston Scientific. Dr. Kalloo is an equity holder for Apollo Endosurgery. Dr. Singh is a consultant for Abbvie and Theraly; an advisory board participant for Cook Medical; and has active grant funding from Orgenesis.

References

[1] Yadav D, Lowenfels AB. The epidemiology of pancreatitis and pancreatic cancer. Gastroenterology 2013; 144: 1252-1261

[2] Anand G, Hutfless SM, Akshintala VS et al. A population-based evaluation of severity and mortality among transferred patients with acute pancreatitis. Pancreas 2014; 43: 1111-1116

[3] Elmunzer B], Scheiman JM, Lehman GA et al. A randomized trial of rectal indomethacin to prevent post-ERCP pancreatitis. N Engl J Med 2012; 366: 1414-1422 
[4] He Q, Wang L, Peng C et al. Modified prophylactic 5-fr pancreatic duct stent enhances the rate of spontaneous dislodgement: A multicenter randomized controlled trial. United European Gastroenterol J 2018; 6: 1519-1526

[5] Geokas MC. Acute pancreatitis. Calif Med 1972; 117: 25-39

[6] Sagi SV, Schmidt S, Fogel E et al. Association of greater intravenous volume infusion with shorter hospitalization for patients with postERCP pancreatitis. J Gastroenterol Hepatol 2014; 29: 1316-1320

[7] Buxbaum J, Yan A, Yeh K et al. Aggressive hydration with lactated Ringer's solution reduces pancreatitis after endoscopic retrograde cholangiopancreatography. Clin Gastroenterol Hepatol 2014; 12: 303-307 e301

[8] Choi JH, Kim H], Lee BU et al. Vigorous periprocedural hydration with lactated ringer's solution reduces the risk of pancreatitis after retrograde cholangiopancreatography in hospitalized patients. Clin Gastroenterol Hepatol 2017; 15: 86-92 e81

[9] Mok SRS, Ho HC, Shah P et al. Lactated Ringer's solution in combination with rectal indomethacin for prevention of post-ERCP pancreatitis and readmission: a prospective randomized, double-blinded, placebo-controlled trial. Gastrointest Endosc 2017; 85: 1005-1013

[10] Kamal A, Akshintala VS, Talukdar R et al. A randomized trial of topical epinephrine and rectal indomethacin for preventing post-endoscopic retrograde cholangiopancreatography pancreatitis in high-risk patients. Am J Gastroenterol 2019; 114: 339-347

[11] Harris PA, Taylor R, Thielke R et al. Research electronic data capture (REDCap)-a metadata-driven methodology and workflow process for providing translational research informatics support. J Biomed Inform 2009; 42: 377-381

[12] Cotton PB, Lehman G, Vennes J et al. Endoscopic sphincterotomy complications and their management: an attempt at consensus. Gastrointest Endosc 1991; 37: 383-393
[13] Andriulli A, Loperfido S, Napolitano G et al. Incidence rates of postERCP complications: a systematic survey of prospective studies. Am J Gastroenterol 2007; 102: 1781-1788

[14] Mallery JS, Baron TH, Dominitz JA et al. Complications of ERCP. Gastrointest Endosc 2003; 57: 633-638

[15] Cheon YK, Cho KB, Watkins JL et al. Efficacy of diclofenac in the prevention of post-ERCP pancreatitis in predominantly high-risk patients: a randomized double-blind prospective trial. Gastrointest Endosc 2007; 66: 1126-1132

[16] DiMagno M], Wamsteker EJ, Maratt J et al. Do larger periprocedural fluid volumes reduce the severity of post-endoscopic retrograde cholangiopancreatography pancreatitis? Pancreas 2014; 43: 642-647

[17] Shaygan-Nejad A, Masjedizadeh AR, Ghavidel A et al. Aggressive hydration with Lactated Ringer's solution as the prophylactic intervention for postendoscopic retrograde cholangiopancreatography pancreatitis: A randomized controlled double-blind clinical trial. J Res Med Sci 2015; 20: 838-843

[18] Smeets X, da Costa DW, Fockens P et al. Fluid hydration to prevent post-ERCP pancreatitis in average- to high-risk patients receiving prophylactic rectal NSAIDs (FLUYT trial): study protocol for a randomized controlled trial. Trials 2018; 19: 207

[19] de-Madaria E, Herrera-Marante I, Gonzalez-Camacho V et al. Fluid resuscitation with lactated Ringer's solution vs normal saline in acute pancreatitis: A triple-blind, randomized, controlled trial. United European Gastroenterol J 2018; 6: 63-72

[20] Wu BU, Hwang JQ, Gardner TH et al. Lactated Ringer's solution reduces systemic inflammation compared with saline in patients with acute pancreatitis. Clin Gastroenterol Hepatol 2011; 9: 710-717 e711

[21] Singh VK. High volume lactated Ringer's for post-endoscopic retrograde cholangiopancreatography pancreatitis prophylaxis: ready for prime time? Clin Gastroenterol Hepatol 2017; 15: 93-95 